\title{
Kimura's disease affecting the axillary lymph nodes: a case report
}

\author{
Kenji Kuroda ${ }^{1,2}$, Shinichiro Kashiwagi ${ }^{2 *}$, Hitoshi Teraoka', Haruhito Kinoshita', Mikio Nanbara', Eiji Noda',
} Takaaki Chikugo ${ }^{3}$, Kosei Hirakawa ${ }^{2}$ and Masaichi Ohira ${ }^{2}$

\begin{abstract}
Background: Kimura's disease (KD; eosinophilic granuloma of soft tissue) is an inflammatory granulomatous disorder of unknown cause with eosinophilic infiltration that occurs mainly in soft tissue. KD occurs mainly in the head and neck, but development in the axillary region is very rare.

Case presentation: A 74-year-old Japanese woman was evaluated for a mass that she noted in the left axillary region. On physical examination, there was a palpable, thumb-sized, hard, elastic, freely movable mass in the left axilla. Blood tests showed elevated soluble interleukin-2 receptor (sIL-2R), normal serum immunoglobulin (Ig) G4, and elevated serum lgE. Ultrasonography of the left axilla showed multiple lymph nodes (LNs) with irregular margins in which central hyperechogenicity was lost. A systemic search by computed tomography (CT) showed no systemic lymphadenopathy or other mass-like lesions suspicious for a primary tumour other than in the left axillary LNs. Biopsy of an excised LN was performed under local anaesthesia for a definitive diagnosis. Histopathology showed various-sized lymphoid follicles, large nodular lesions with an enlarged mantle zone, multiple various-sized germinal centres in single nodules, and eosinophilic infiltration between the nodes. Immunohistochemical $(\mathrm{IHC})$ staining of the germinal centres was positive for cluster of differentiation (CD) 10, positive for B-cell lymphoma (bcl)-6, and negative for bcl-2. These findings led to a diagnosis of KD. Ultrasound after 3 months of follow-up showed disappearance of the axillary lymphadenopathy.
\end{abstract}

Conclusions: A very rare case of KD in the axillary LNs was described. KD has the potential to occur in any region.

Keywords: Kimura's disease, Axillary lymph nodes, Eosinophilic granuloma, IgE-RIST, Biopsy

\section{Background}

Kimura's disease (KD; eosinophilic granuloma of soft tissue) is an inflammatory granulomatous disorder of unknown cause with eosinophilic infiltration that occurs mainly in soft tissue. KD presents clinically as pruritic and pigmented lymph nodes (LNs) or painless masses in soft tissue [1]. KD occurs mainly in the head and neck, but development in the axillary region is very rare [2]. This report describes a patient with KD in the axillary along with a review of the relevant literature.

\section{Case presentation}

A 74-year-old Japanese woman was evaluated for a mass that she noted in the left axillary region. Her past and medication histories were unremarkable. On physical

\footnotetext{
*Correspondence: spqv9ke9@view.ocn.ne.jp

2Department of Surgical Oncology, Osaka City University Graduate School of

Medicine, 1-4-3 Asahi-machi, Abeno-ku, Osaka, Japan

Full list of author information is available at the end of the article
}

examination, there was a palpable, thumb-sized, hard, elastic, freely movable mass in the left axilla. No breast masses or skin lesions were noted. Blood tests showed a normal white blood cell count, but a differential showed mildly elevated eosinophils (6\%). Biochemical tests showed mildly elevated alkaline phosphatase (ALP) of 326 IU/l (normal, 60-200 IU/l), carcinoembryonic antigen (CEA) of $2.3 \mathrm{ng} / \mathrm{ml}$ (normal, $<5.0 \mathrm{ng} / \mathrm{ml}$ ), cancer antigen 15-3 (CA15-3) of $10.0 \mathrm{IU} / \mathrm{ml}$ (normal, $<30.0 \mathrm{IU} / \mathrm{ml}$ ), National Cancer Center-stomach-439 (NCC-ST-439) of $3.3 \mathrm{U} / \mathrm{ml}$ (normal, $<7.0 \mathrm{U} / \mathrm{ml}$ ), and elevated soluble interleukin-2 receptor (sIL-2R) of $604.9 \mathrm{U} / \mathrm{ml}$ (normal, 122.0-496.0 U/ml). Serum immunoglobulin (Ig) G4 was normal at $47.7 \mathrm{mg} / \mathrm{dl}$ (normal, $4.8-105.0 \mathrm{mg} / \mathrm{dl}$ ), but immunoglobulin E-radioimmunosorbent test (IgE-RIST) results were elevated at $3032 \mathrm{IU} / \mathrm{ml}$ (normal, $<173.0 \mathrm{IU} / \mathrm{ml}$ ).

Ultrasonography (US) of the left axilla showed multiple LNs with irregular margins in which central hyperechogenicity was lost (maximum size: $2.2 \mathrm{~cm} \times 1.5 \mathrm{~cm}$ ) (Fig. 1a, b). 

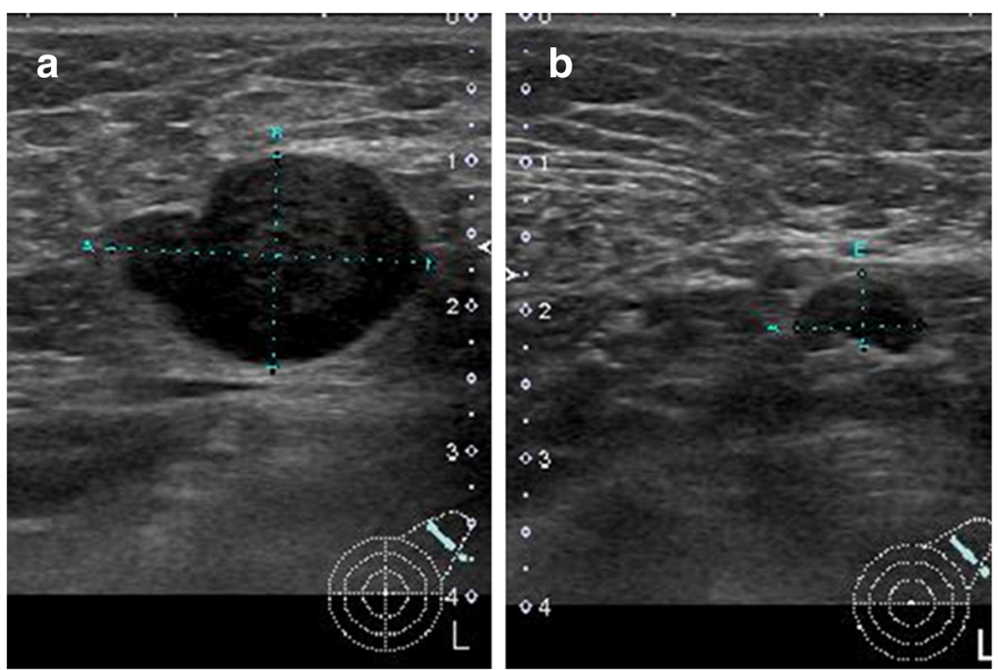

Fig. 1 Ultrasound findings: Ultrasound of the left axilla showed multiple LNs with irregular margins in which central hyperechogenicity was lost (maximum size: $2.2 \mathrm{~cm} \times 1.5 \mathrm{~cm}$; $\mathbf{a}, \mathbf{b}$ )

A systemic search using computed tomography (CT) showed multiple lymphadenopathy only in the left axilla, with no other systemic lymphadenopathy or masslike lesions suspicious of a primary tumour (Fig. 2a, b). To differentiate a malignancy such as malignant lymphoma or LN metastases from an unknown primary tumour, a core needle biopsy (CNB) of the left axillary LNs was performed. Pathology examination of the CNB showed LNs with rich eosinophilic infiltration but no evidence of any atypical cells (Fig. 3a, b).

Immunohistochemical (IHC) staining showed no evidence of malignant lymphoma or LN metastases from an occult breast cancer (cytokeratin (CK) 7-negative, CK20-negative, and cluster of differentiation (CD) 117negative). Biopsy of an excised LN was performed under local anaesthesia for a definitive diagnosis. Histopathology showed various-sized lymphoid follicles, large nodular lesions with an enlarged mantle zone, and multiple germinal centres in single nodules (Fig. 4a). Proliferation of small blood vessels and marked eosinophilic infiltration were also observed between the nodes (Fig. 4b). IHC staining of the germinal centres was positive for $\mathrm{CD} 10$, negative for B-cell lymphoma (bcl)-2, and positive for bcl-6 (Fig. 4c-e). Staining was positive for IgG in the germinal centres and between nodes, and some areas were IgG4-positive (Fig. 4f).

An IgG4-related disease was also considered, but serum IgG4 levels were normal. The findings eventually led to a diagnosis of KD. The patient was followed up, in part based on her own wishes, and an ultrasound performed 3 months later showed disappearance of the axillary lymphadenopathy. As of this writing, 6 months after the initial examination, there has been no recurrence of $K D$.

\section{Discussion}

$\mathrm{KD}$ is a chronic inflammatory granulomatous disorder with the formation of masses characterized by eosinophilic infiltration and lymphoid follicular hyperplasia in subcutaneous soft tissue. KD was first reported by Kimura et al. in 1948 [3]. KD has a predilection for Asian males in their 30s and 40s and presents clinically as single or multiple masses in subcutaneous soft tissue and LNs; although the masses are often painless, they
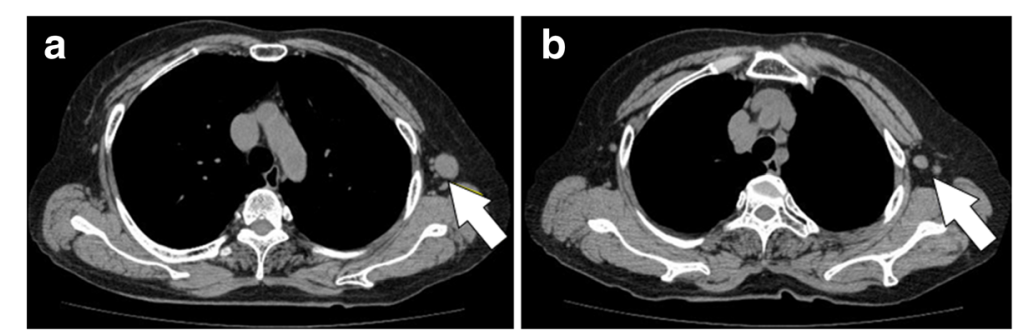

Fig. 2 CT image findings: A systemic search using computed tomography $(C T)$ showed multiple lymphadenopathy only in the left axilla, with no other systemic lymphadenopathy or mass-like lesions suspicious of a primary tumour $(\mathbf{a}, \mathbf{b})$ 


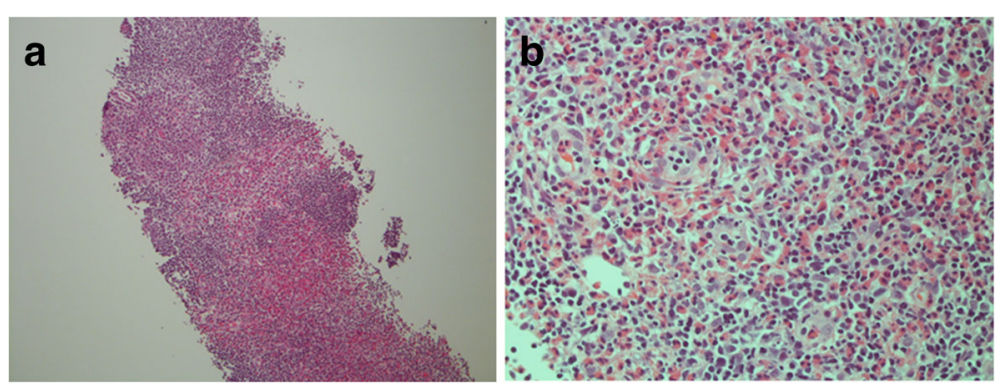

Fig. 3 Pathological findings of core needle biopsy: Pathological examination of the biopsy specimens showed LNs with rich eosinophilic infiltration, but no evidence of any atypical cells $(\mathbf{a}, \times 40 ; \mathbf{b}, \times 400)$

may be associated with pruritus and skin pigmentation [2]. These masses mostly occur in the head and neck, and although the limbs and inguinal regions are sometimes affected, occurrence in the axillary region is rare $[2,4,5]$. Additionally, there are case reports that KD involving the thoracic and abdominal lymph nodes may be confused with malignant lymphoma or other metastatic cancers $[6,7]$. Therefore, KD has the potential to occur in any region.

The blood tests used to diagnose KD typically show peripheral blood eosinophilia and elevated serum IgE levels [1]. Histopathology shows various-sized germinal centres with lymphoid follicular hyperplasia, surrounding eosinophilic infiltrates, and blood vessels with vascular endothelial cell swelling. IHC staining is often positive for IgE in the germinal centres of follicles [8-10].

The present patient was an elderly woman who initially presented with enlarged axillary LNs. However, considering the rarity of $\mathrm{KD}$ and its epidemiologic features, it was not initially suspected. The differential diagnosis of lymphadenopathy included LN metastases due to occult breast cancer, malignant lymphoma, reactive lymphadenitis, IgG4-related disease, and eosinophilic granuloma. Therefore, a detailed pathological examination was performed to make a definitive diagnosis. Haematoxylin-eosin (HE) staining showed lymphoid follicular hyperplasia suggesting progressively transformed germinal centres and germinal centres of varying sizes with eosinophilic infiltration between the follicles. The morphologic features suggested KD or IgG4-related disease, but the blood test results showing mild peripheral blood eosinophilia, elevated serum IgE, and normal serum IgG4 ultimately led to a diagnosis of KD.

No definitive therapy for KD has been established, but the use of surgical excision, radiation therapy, or drug treatment alone or in combination has been reported. Surgery is usually performed if the masses can be excised, but other treatment may be selected in patients with possible neurovascular injury or masses that are not localized [11]. Radiation therapy is also associated with a relatively low rate of disease

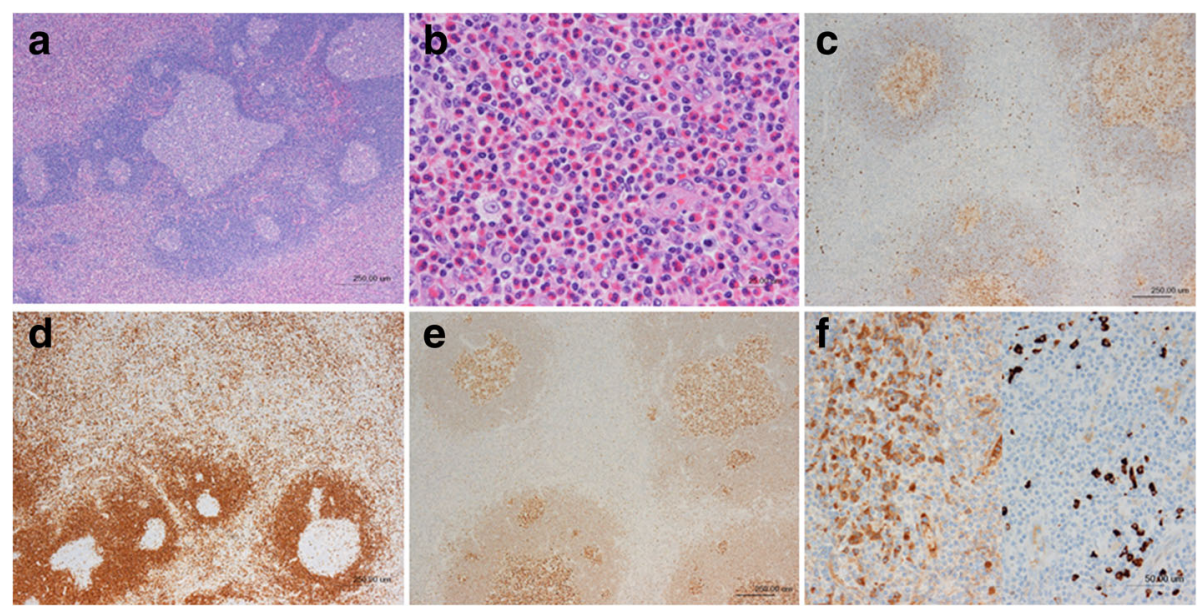

Fig. 4 Pathological findings of the excised lymph nodes: Histopathology showed various-sized lymphoid follicles and large nodular lesions with an enlarged mantle zone, and multiple germinal centres in single nodules (a). Proliferation of small blood vessels and marked eosinophilic infiltration were also observed between the nodes (b). Immunohistochemical staining of the germinal centres was positive for CD10 (c), negative for bcl-2 (d), and positive for bcl-6 (e). Staining was positive for lgG in the germinal centres and between nodes, and some areas were lgG4-positive (f) 
recurrence, but adverse reactions may occur, and it might not be indicated for younger patients.

Drug therapy includes steroids, non-steroidal antiinflammatory and analgesic drugs, and suplatast tosilate (Th2 cytokine inhibitor), but disease recurrence rates are high, and the risk of adverse reactions with long-term use must be considered [11]. The present patient underwent $\mathrm{LN}$ excision for a definitive diagnosis, and after a follow-up period, the axillary lymphadenopathy disappeared. However, because KD has a high recurrence rate, long-term follow-up is required.

\section{Conclusions}

A very rare case of $K D$ in the axillary lymph nodes was described. KD has the potential to occur in any region.

\section{Abbreviations}

ALP: Alkaline phosphatase; bcl: B-cell lymphoma; CA15-3: Cancer antigen 15-3; CD: Cluster of differentiation; CEA: Carcinoembryonic antigen; CK: Cytokeratin; CNB: Core needle biopsy; CT: Computed tomography; Ig: Immunoglobulin; IgERIST: Immunoglobulin E-radioimmunosorbent test; IHC: Immunohistochemical; KD: Kimura's disease; LN: Lymph node; NCC-ST-439: National Cancer Centerstomach-439; sIL-2R: soluble interleukin-2 receptor; US: ultrasonography

\section{Acknowledgements}

We thank Hirokazu Nakamine (Department of Laboratory Medicine, Japan Baptist Hospital) for helpful advice regarding the pathological evaluation. This report was supported in part by Grants-in Aid for Scientific Research (KAKENHI, Nos. 25461992 and 26461957) from the Ministry of Education, Science, Sports, Culture and Technology of Japan.

\section{Funding}

This study was supported in part by Grants-in Aid for Scientific Research (KAKENHI, Nos. 25,461,992 and 26,461,957) from the Ministry of Education, Science, Sports, Culture and Technology of Japan.

\section{Availability of data and materials}

The datasets supporting the conclusions of this article are included within the article.

\section{Authors' contributions}

All the authors were involved in the preparation of this manuscript. KK collected the data and wrote the manuscript. SK, HT, HK, MN, and EN performed the operation and designed the study. TC performed pathological diagnosis. KK and SK summarized the data and revised the manuscript. $\mathrm{KH}$ and $\mathrm{MO}$ made substantial contributions to the study design, performed the operation, and revised the manuscript. All the authors read and approved the final manuscript.

\section{Competing interests}

The authors declare that they have no competing interests.

\section{Consent for publication}

Written informed consent was obtained from the patient for the publication of this case report and any accompanying images. A copy of the written consent form is available for review by the editor-in-chief of this journal.

\section{Ethics approval and consent to participate}

Not applicable.

\section{Publisher's Note}

Springer Nature remains neutral with regard to jurisdictional claims in published maps and institutional affiliations.

\section{Author details}

'Department of Surgery, Baba Memorial Hospital, Higashi 4-244 Hamadera Funao-cho, Nishi-ku, Sakai, Osaka 592-8555, Japan. ${ }^{2}$ Department of Surgical Oncology, Osaka City University Graduate School of Medicine, 1-4-3 Asahi-machi, Abeno-ku, Osaka, Japan. ${ }^{3}$ Department of Pathology, Kinki University Faculty of Medicine, Osaka-sayama, Osaka 589-8511, Japan.

Received: 16 January 2017 Accepted: 21 May 2017

Published online: 26 May 2017

\section{References}

1. Wang DY, Mao JH, Zhang Y, et al. Kimura disease: a case report and review of the Chinese literature. Nephron Clin Pract. 2009;111:c55-61.

2. Abuel-Haija M, Hurford MT. Kimura disease. Arch Pathol Lab Med. 2007; 131:650-1.

3. Kimura T, Yoshimura S, Ishikawa E. On the unusual granula- tion combined with hyperplastic changes of lymphatic tissue. Trans Soc Pathol Jpn. 1948; 37:179-80.

4. Kakkar A, Gupta RK, Khanna P, Balasundaram P, Ray R, Shukla NK. Kimura disease of the breast - a previously Undescribed entity. Breast J. 2016;22:456-9.

5. Nag VK, Nandan D, Bhardwaj M. Kimura's disease presenting with inguinal lymphadenopathy in an 11-year old girl: a case report. Trop Dr. 2015:45:54-6.

6. Lee S, Jung SJ, Park SK, Kang KP, Jang KY, Kang MJ, et al. Kimura's disease involving thoracic and abdominal lymph nodes in a Hemodialysis patient. Korean J Intern Med. 2005;20:159-62.

7. Madhavan $\mathrm{M}$, Othman $\mathrm{NH}$, Singh MS, Indudharan $\mathrm{R}$, Sharma HS, Shamsuddin AR. Kimura's disease: a report of three cases with a brief review of literature. Acta Otorhinolaryngol Ital. 2000;20:284-9.

8. Hui PK, Chan JK, Ng CS, Kung IT, Gwi E. Lymphadenopathy of Kimura's disease. Am J Surg Pathol. 1989;13:177-86.

9. Urabe A, Tsuneyoshi M, Enjoji M. Epithelioid hemangioma versus Kimura's disease. A comparative clinicopathologic study. Am J Surg Pathol. 1987; 11(10):758-66.

10. Takeishi M, Makino Y, Nishioka H, Miyawaki T, Kurihara K. Kimura disease: diagnostic imaging findings and surgical treatment. J Craniofac Surg. 2007; 18:1062-7.

11. Tsukagoshi H, Nagashima M, Horie T, Oyama T, Yoshii A, Sato T, et al. Kimura's disease associated with bronchial asthma presenting eosinophilia and hyperimmunoglobulinemia E which were attenuated by suplatast tosilate (IPD-1151T). Intern Med. 1998:37(12):1064-7.

Submit your next manuscript to BioMed Central and we will help you at every step:

- We accept pre-submission inquiries

- Our selector tool helps you to find the most relevant journal

- We provide round the clock customer support

- Convenient online submission

- Thorough peer review

- Inclusion in PubMed and all major indexing services

- Maximum visibility for your research

Submit your manuscript at www.biomedcentral.com/submit
Biomed Central 